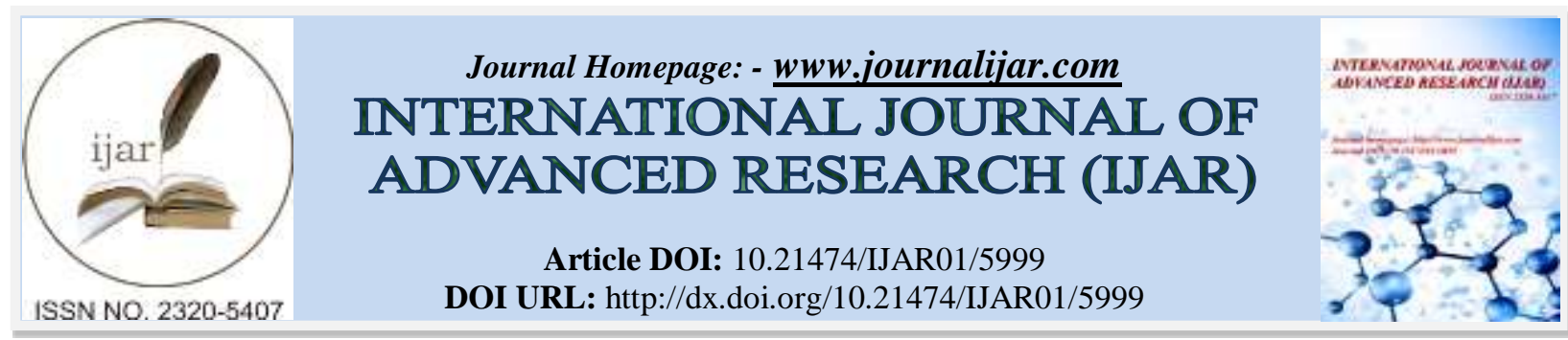

RESEARCH ARTICLE

\title{
ROLE OF THIAMINE DEFICIENCY AND EFFICACY OF THIAMINE IN TREATMENT OF PATIENTS WITH HEART FAILURE.
}

\author{
${ }^{*}$ Dr. Shamim Iqbal ${ }^{1}$, Dr. Tariq Abdullah ${ }^{2}$, Dr. Nuzhat ${ }^{3}$, Dr. Khurshid Iqbal ${ }^{4}$ and Dr. Mir Ishaq ${ }^{5}$. \\ 1. Consultant Cardiologist, MMABM Hospital, Anantnag. \\ 2. Consultant Physician, MMABM Hospital, Anantnag. \\ 3. Medical Officer, Health \& Medical Education, J\&K. \\ 4. Ex-Professor and Head, Department of Cardiology, SKIMS. \\ 5. General Practitioner.
}

\section{Manuscript Info}

Abstract

Manuscript History

Received: 07 October 2017

Final Accepted: 09 November 2017

Published: December 2017

Copy Right, IJAR, 2017,. All rights reserved.

\section{Introduction:-}

Thiamine is a water soluble B-complex vitamin readily available in cereal grains, yeast, nuts and meat products. It combines with adenosine triphosphate in liver, kidneys and leukocyte to form thiamine pyrophosphate, a coenzyme in carbohydrate metabolism ${ }^{1}$.

Diagnosis of thiamine deficiency is frequently obtained by measuring thiamine pyrophosphate effect (TPPE). TPPE reflects thiamine concentrations on the basis of activity of thiamine diphosphate on the transketolase enzyme of pentose shunt ${ }^{2}$.

A thiamine pyrophosphate effect of 0 to $15 \%$ is considered normal, $15 \%$ to $24 \%$ indicative of marginal deficiency, and $>25 \%$ associated with several deficiency ${ }^{3}$.

Thiamine status can be assessed also by direct measurement of thiamine pyrophosphate in erythrocytes or whole blood by high performance liquid chromatography (HPLC). This method is precise and yields results similar to erythrocyte activation assay ${ }^{4}$.

Specific signs and symptoms observed in thiamine-deficient patients represent alterations in the biochemistry of thiamine that result in impaired oxidative phosphorylation, increased levels of proximal metabolites (pyruvate), and decreased transketolase activity in RBCs, liver, heart and other organs. Thiamine deficiency manifests as dry or wet beri beri. Dry beri beri involves peripheral neuropathy that can be permanent even following thiamine repletion. Neurological side effects of thiamine deficiency can progress to Wernicke-koraskoff syndrome ${ }^{5}$.

Heart failure (HF) is a common, disabling and costly disease throughout the world. The recommended pharmacological therapy includes the use of angiotensin converting enzyme inhibitors, angiotensin receptor 
blockers, diuretics, beta-blockers and aldosterone antagonists. Additional non-pharmacological measures, such as cardiac resynchronization therapy, implantable cardioverter defibrillators, and exercise training have shown beneficial outcome sin quality of life, morbidity, and/or mortality of HF patients ${ }^{6}$.

Although current therapies have addressed hemodynamics, neurohormonal modulation and electrophysiological aspects of HF, these therapies have not targeted the metabolic needs of the failing heart ${ }^{7}$.

The heart has been described as a metabolic omnivore, because it has the capacity to oxidize fat and carbohydrate simultaneously or interchangeably for its energy needs ${ }^{8}$.

The impact of heart failure and its treatment on specific nutrient requirements is unknown. Furthermore, depletion of water-soluble B-vitamins that play key roles in the production of cellular energy in patients with heart failure can contribute to depletion of energy in failing heart ${ }^{9}$.

Patients with New York Heart Association (NYHA) Class III/IV heart failure appear to have more severe thiamine deficiency than their class I/II counterparts. Frusemide, commonly used in such patients may exacerbate this problem through urinary excretion of thiamine. There also appears to be an increased need for thiamine in the setting of heart failure (HF), as similar nutritional amounts are inadequate in HF patients compared with controls ${ }^{10}$.

Inadequate caloric and protein intake is common in HF patients and the diets of patients with heart failure do not contain adequate amounts of thiamine-rich foods ${ }^{10,11}$.

Given the proposed role of thiamine deficiency in heart failure patients, we conducted this study to assess the thiamine status in a subset of heart failure patients and also for the assessment of clinical and hemodynamic effects of thiamine repletion in these patients.

\section{Material and Methods:-}

After obtaining the ethical clearance from the Institutional Ethical Committee, the present study was conducted in the Department of Cardiology, SKIMS Srinagar. A total of 50 patients were enrolled in this study.

\section{Inclusion Criteria:-}

All patients with chronic CHF and New York Heart Association (NYHA) functional Class 2 and 4 due to (i) chronic ischemic heart disease, (ii) idiopathic dilated cardiomyopathy, (iii) valvular heart disease, (iv) atrial fibrillation were included in the study.

\section{Exclusion Criteria:-}

1. Acute myocardial infarction.

2. $\quad$ LVEF $>45 \%$

3. Recent thiamine or multivitamin supplements

$4 \quad$ Identifiable (by history and physical examination)

All the included patients were admitted in the Cardiology Department with the diagnosis of heart failure made using Framingham criteria ${ }^{12}$ requiring the presence of two major or one major and two minor criteria.

The study participants were randomly allocated to one week of inpatient double blind intravenous therapy with either placebo or thiamine given as two daily I/V doses of either normal saline or 100mg thiamine Hcl. Each patient underwent two dimensional echocardiographic examination before the intravenous treatment and at the end of inpatient week using a commercially available system.

Thiamine system was evaluated by measuring thiamine pyrophosphate effect (TPPE) or erythrocyte transketolase activity prior to and at the end of the week of intravenous treatment by semi-automatic method adopted by JN Mount et $\mathrm{al}^{13}$. In addition to thiamine status, the following clinical parameters were daily assessed for 1 week: (i) blood pressure, (ii) heart rate (iii) body weight and (iv) 24 hour urinary output. 
Further, NYHA class, complete blood count, serum glucose, serum $\mathrm{Na}^{+} / \mathrm{K}^{+}$, blood urea, serum creatinine and urine analysis were compared before and after one week of treatment. All patients who were taking drugs 5 days before entering the hospital for the study continued them at the same dosages throughout the full 7 weeks of the study.

\section{Statistical Methods:-}

Standard statistical procedure were used to analyze the data. Date was described as mean+SD and percentages. Inter-group comparison was done by Mann-Whitney ' $U$ ' test and intra-group variance was measured by Wilcoxon signed rank test at 95\% CI. Statistical package for social sciences (SPSS 18) and Microsoft Excel software were used for data analysis. A p value of $<0.005$ was taken as statistically significant.

\section{Observations And Results:-}

Males constituted 52\% and the females $48 \%$ in the thiamine group while as in the placebo group they comprised $56 \%$ and $44 \%$ respectively with a non-significant $\mathrm{p}$ value between them. Similarly the mean age in the thiamine group was $61.4+7.1$ years and in the placebo group $62.4+8.0$ years.

The etiology of heart failure in the thiamine group was idiopathic dilated cardiomyopathy (68\%), ischemic heart disease (20\%) and valvular heart disease (12\%) while as in the placebo group it was $60 \%, 24 \%$ and $16 \%$ respectively with insignificant $p$ value between the two groups.

There was no significant difference in baseline characteristics between the two treatment groups. Further there were no gross abnormalities in the hemogram, serum chemistry and urine analysis at baseline and at 1 week in both treatment groups.

In the two treatment groups, the percentage of patients who were on $>40 \mathrm{mg} / \mathrm{day}$ of furosemide was $52 \%$ and $60 \%$ with a non-significant statistical difference. The baseline TPPE in the group receiving <40mg/day of furosemide or who were not receiving any furosemide was $15.1+5.1 \%$ with a significant $\mathrm{p}$ value between the two groups.

There was a statistically significant increase in both systolic and diastolic blood pressure of around $10 \mathrm{mmHg}$ and a statistically significant decrease in heart rate of about $16 / \mathrm{min}$ after one week of treatment in the thiamine group. No significant change was observed after one week of treatment in systolic blood pressure, diastolic blood pressure and heart rate in the place group.

There was statistically significant decrease of $3 \mathrm{~kg}$ in weight after one week of treatment in the thiamine group while as no significant change occurred in the placebo group. A significant change in weight was observed in the thiamine group compared to placebo group with a p value of $<0.001$.

Urinary output was an increase of about $700 \mathrm{ml}$ per day in the thiamine group while as there was no significant change in the placebo group. The difference between $3^{\text {rd }}$ day urinary output in thiamine and placebo group was significant with a $\mathrm{p}$ value of $<0.001$.

Significant improvement in New York Heart Association Class from 2.2 to 1.6 was observed in thiamine group with no significant change in the placebo group.

There was a significant increase in LVEF at both 1 and 7 weeks in thiamine group while as in placebo group, no significant change was found at 1 week while as at 7 weeks there was a significant increase in LVEF.

A statistically significant $7 \%$ increase in LVEF in thiamine group compared to $0.7 \%$ in placebo group (p value < 0.001 ) at one week while as at 7 weeks after giving oral thiamine for 6 weeks in both treatment arms there was an increase of $18.4+9.0 \%$ and $19.4+7.2 \%$ in LVEF in the thiamine and placebo groups respectively with a nonsignificant difference between the two.

There was a significant decrease in TPPE in thiamine group from $16.4 \%$ to $6.8 \%(\mathrm{p}<0.001)$ at one week while as in the placebo group no significant change was observed at one week. The change in TPPE at one week in thiamine group was statistically significant compared to placebo group. 


\section{Discussion:-}

In this hospital based study, heart failure patients with diverse etiologies were recruited for evaluation of thiamine status and the role of thiamine, if any, in the amelioration of symptoms and the impact on various clinical and laboratory parameters.

In the thiamine treatment group both the systolic and diastolic blood pressure increased by an average of $10 \mathrm{mmHg}$ and the heart rate decreased by average 16 beats/min (both statistically significant) while as there was no significant change in these parameters in the group receiving placebo treatment. This is consistent with other studies ${ }^{14,13}$. However Ilam Shimon et al ${ }^{15}$ could not demonstrate the increase in blood pressure and decrease in heart rate in their study in the thiamine treatment group attributing in to severe additional cardiac pathology in their patients in this group

In the thiamine treatment group, there was a statistically significant decrease of about $3 \mathrm{~kg}$ of weight after one week of treatment and significant increase of about $700 \mathrm{ml} /$ day urinary output on $3^{\text {rd }}$ day of treatment which is attributed to the natriuretic effect of thiamine. Hanna Seligmann et $\mathrm{al}^{13}$ demonstrated a mean increase of urinary output of $500 \mathrm{ml} / 24$ hours with an average weight loss of $1 \mathrm{~kg}$ in thiamine treatment group. Brisk diuresis was similarly shown by Ilan Shimon et $\mathrm{al}^{15}$ in their thiamine treatment cohort of heart failure patients.

There was a statistically significant increase of about $7 \%$ in LVEF at one week in the thiamine treatment group while as no significant change was demonstrated in the LVEF of placebo treatment group. This correspondingly translated into improvement in functional class from mean NYHA class 2.2 to $1.6(\mathrm{p}<0.001)$ in the thiamine group. In the placebo group the mean NYHA class had an insignificant decrease from .2 to 2.1 (p 0.157).

After 6 weeks of oral thiamine replacement in both treatment arms, there was about $20 \%$ increase in LVEF in both the groups. This was a significant finding because an increase in ejection fraction has been associated with a favourable effect on survival in patients with congestive heart failure ${ }^{1}$. Thiamine deficient heart disease is characterized by sodium and water retention, peripheral vasodilation and myocardial failure as a result thiamine deficiency is presumed to worsen symptoms in the setting of established congestive heart failure ${ }^{10}$.

The improvement in LVEF after thiamine replacement has been consistently demonstrated in other studies ${ }^{13,15}$. Shimon I et $\mathrm{al}^{15}$ demonstrated an increased in LVEF of $22 \%$ in 27 patients of heart failure who were put on 6 weeks of oral thiamine treatment in their study.

There was a significant decrease in TPPE in thiamine-treated patients from $16.4 \%$ to $6.8 \%$ (p < 0.001$)$ after one week of treatment but no significant change in TPPE at one week in the placebo group and the change in TPPE at one week in thiamine group was statistically significant compared to placebo group ( $p<0.001)$. Since the baseline TPPE was similar in both the treatment arms, it implies that there was subclinical thiamine deficiency which got corrected in the thiamine treatment group. The thiamine deficiency in heart failure patients has been observed by Stacy A. Hanninen et $\mathrm{al}^{10}, \mathrm{H}$. Seligmann et $\mathrm{al}^{24}$ and I. Shimon et $\mathrm{al}^{15}$ in their studies; however the patient cohort enrolled in these studies were on high dose diuretics. Yiu et al ${ }^{16}$ first described increased loses of thiamine secondary to loop diuretic therapy. Since then several other studies ${ }^{10,13,17}$ have replicated these findings suggesting that loop diuretic therapy can lead to thiamine deficiency mainly secondary to hyperexcretion.

However, among the subset of heart failure patients recruited in our study, approximately only one half of patients was on furosemide and the dosage of furosemide $(>40 \mathrm{mg} /$ day) used as a criterion in our study was significantly lower than the dose of furosemide taken as eligibility criterion in other studies ${ }^{13,15}$.

The issue of age as a factor contributing to the prevalence of thaimien deficiency was evaluated by Levy et al ${ }^{17}$ who conducted a prospective trial of patients with a mean age of $47+10$ years, NYHA class of $2.5+0.6$ and mean LVEF of $22+9 \%$. No evidence of thiamine deficiency was fund in their population of heart failure patients.

\section{Conclusion:-}

The metabolic and hemodynamic features of the failing heart can be further aggravated by thiamine deficiency. It is thus possible that in patients with advanced $\mathrm{CHF}$, thiamine deficiency is one determinant of their poor clinical 
status. The importance of recognizing this somewhat neglected possible association cannot be over-emphasized since thiamine deficiency induced heart failure is preventable and curable.

\section{Bibliography:-}

1. Leslie D and Gherghiade M. Is there a role for thiamine supplementation in management of heart failure. Am Heart J 1996; 131: 1248-50.

2. Baines M, Davies G. The evaluation of erythrocyte thiamine diphosphate as an indicator of thiamine status in man, and its comparison with erythrocyte transketolase activity measurements. Ann Clin Biochem 1988; 25: 698-705.

3. Hass RH. Thiamine and the brain. Ann Rev Nutr 1988; 8: 483-515.

4. Dinesh Talwar, Helen Davidson, Josephine Cooney and Denis Reilly. Vitamin B1 status assessed by direct measurement of thiamine pyrophosphate in erythrocytes or whole blood by HPLC: comparison with erythrocyte transketolase activation assay. Clinical Chemistry 2000; 46(5): 704-710.

5. Wooley JA. Characteristics of thiamine and its relevance to the management of heart failure. Nutr Clin Pract 2008; 23: 487.

6. Hunt SA, Abraham WT, Chin MH et al. Focused update incorporated into the ACC/AHA 2005 guidelines for the diagnosis and management of heart failure in adults. J Am Coll Cardiol 2009; 53 ed. 90.

7. Taegtmeyer H, Razeghi, Young ME. Alterations in cardiac metabolism in heart failure. Philadelphia, PA Saunders, 2004.

8. Taegtmever H. Carbohydrate inter-conversion and energy production. Circulation 1985; 72(IV): 1-8.

9. Keith ME, Walsh NA, Darling PB et al. B vitamin deficiency in hospitalized patients with heart failure. Journal of American Dietetic Association 2009 Aug; 108 (8).

10. Hanninen SA, Darling PB, Sole MJ et al. The prevalence of thiamine deficiency in hospitalized patients with congestive heart failure. J Am Coll Cardiol 2006; 47: 354-61.

11. Kwok T, Falconer-Smith JF, Potter JF, Ives DR. Thaimine status of elderly patients with cardiac failure. Age Ageing 1992; 21: 67-71.

12. Ho KKL et al. Framingham Criteria for diagnosis of congestive heart failure. Circulation 1993; 88: 107.

13. Hanna S, Hillel H, Shmuel R, Natan K, Rachel T, Michael M, Zvi V, David E. Thiamine deficiency in patients with congestive heart failure receiving long term furosemide therapy: a pilot study. Am J Med 1991; 91: 15155 .

14. Freye E, Hartung E. The potential use of thiamine in patients with cardiac insufficiency. Acta Vitaminol Enzymol 1982; 4(4): 285-90.

15. Shimon I, Almog $\mathrm{S}$, Vered $\mathrm{Z}$ et al. Improved left ventricular function after thiamine supplementation in patients with congestive heart failure receiving long term furosemide therapy. Am J Med 1995; 98: 485-90.

16. Yui Y, Itokawa Y, Kawai C. Furosemide-induced thiamine deficiency. Cardiovasc Res 1980; 14: 537-40.

17. Levy WC, Soine LA et al. Thiamine deficiency in congestive heart failure (Letter). Am J Med 1992; 93: 705 06. 